\title{
Research on Legal Nature of Domain Name Infringement in International Internet
}

\author{
Zhenyuan $\mathrm{Du}^{1, \mathrm{a}^{*}}$ \\ ${ }^{1}$ Nanjing university of Chinese medicine, Nanjing 210046, China \\ alawduzy@126.com
}

Keywords: Domain name; Domain name infringement; Intellectual property rights

\begin{abstract}
Domain name infringement is a hot issue in the field of Internet e-commerce. The legal status and legal attributes of domain names are not unified, which is the focus of controversy in the academic circle. Analysis of the characteristics of intellectual property rights and legal characteristics of domain name, domain name is considered to have independent legal status, have the basic characteristics of intellectual property rights. Domain name right is a new type of intellectual property rights, which is a breakthrough and development of the regional, time, uniqueness and proprietary of the traditional intellectual property rights.
\end{abstract}

\section{Introduction}

Internet Domain Name is a kind of certain sign on the Internet corresponding to network protocol address in computers. It is a data string to represent and determine certain numerical address in international Internet formed by a group of letters, numbers, signs etc. (Chen Jiang, \&Deng Jiong, 2000)In essence, domain name has functions of address and sign, thus it has enormous commercial value and advertising effects and has become one of new properties in modern society. However, without explicit and independent definition of domain name, its legal status and nature remain controversies, which has influenced in development of electronic commerce and caused conflicts between domain name rights and rights of name, trademark, trade name, and the domain name rights, which caused tremendous shock to traditional intellectual property system.

\section{The Dispute on Legal Nature of Domain Name}

Domain name is a new concept which legal nature hasn't been defined explicitly in China. The independence of domain name (whether there is a domain name right or not) and whether it is a new intellectual property or not are the hot topics in academic circles and also are the most important problems to clear up before discussing whether domain name should be under the law protection or not and how to protect in law.

The Legal Status Of The Domain Name. There are two main different views on whether the domain name is independent or not. One view is that domain name has no independent value. It is just a technical parameter, or network tools and address code.(Tang Guang-Liang,2012) This view focuses on the technical characteristics of domain names and underestimate the commercial value of domain names. The opposite view is that from the identity characteristics, users have exclusive control over the domain name, that is domain name rights,(Liu Jin-Lin,2002) after they have been audited by the domain name management agency and domain name has an independent legal status and value. In this view, for the domain name rights and attributes, academics are divided into two different views that is the domain name is intellectual property rights or a civil right.

The author believes that in the field of e-commerce, the role of domain name is not just a technical means. The typical case, such as “360 company v. 360 credit loan network", Someone into the "http://www.360daidai.com" website, which prominently at the top left of the "360 loan lending network" logo, and the logo of the figure and number 360 are green, the use of advertising language 
is "do the safest investment platform" and so on, thinking that the P2P platform is the 360 company's financial products, and then intend to invest 5 million.In fact, the "360 Loan Network" is the actual operator of Beijing joint investment financial information service Co., Ltd. which has nothing to do with the 360 companies.It marks the identity of individuals and businesses on the Internet and thus bring large commercial value. Therefore, domain name has independent value and should be regarded as an independent new rights. Its holders should enjoy a certain independent interests, namely domain name rights.

The Legal Nature of Domain Name Rights. The theoretical circles form two viewpoints in respect of the nature of the domain name rights: some one hold that it is the prevailing intellectual property rights, considering the domain name as a new intellectual property with the independent value; the others deem that it is part of civil rights and the essence of domain name is merely the identifier with the function of technical parameter, which is one kind of name rights belonging to civil rights rather than intellectual property.

After analyzing based on the characteristics of intellectual property rights and features of domain name, the author thinks that the domain name has the essential characteristics of intellectual property rights and should be incorporated into the system of intellectual property protection.

First of all, the domain name is a combination of network business identifiers and the websites like the harvest of intellectual labor of the trademark and patent. In addition, in order to increase the network hits and expand the attraction, the domain name's arrangement form needs to be original, in which contains certain intellectual labor. Therefore, domain has the characteristics of invisibility property;

Secondly, the domain name has a special attribute. If one domain name is approved and belongs to the registrant, nobody can register the same one. And intellectual property is also belonged to the registrant, and no one can use it without the consent of the registrant. From this point of view, the attribute of intellectual property existing in the domain name is particularly obvious.

Thirdly, the commercial value of domain name will be difficult to estimate when it is known to the public.And the domain name will become the propellant of enterprise growth, help enterprise hold advantage in the intense market and can bring huge economic benefits for proprietors.

\section{Breakthrough in The Domain Name Right to The Traditional Intellectual Property Rights}

The domain name rights is a kind of new intellectual property rights having the following characteristics compared with the traditional intellectual property rights: First is the breakthrough in region. As the Internet is global, domain name is recognized worldwide as soon as they are registered on the Internet. Access to the same site can be accessed to using the same domain name. Thus, the regional character of domain name breaks through the restriction of traditional country and region, and has been international. While the traditional intellectual property right applies in the certain geographical scope. Its effectiveness is limited to the territory of the country, and in another country it may not be effectively recognized by law.

Second is the breakthrough in time. The validity of the domain name begins with registration. Once the registration is successful, as long as the holder of the domain name pays a certain fee on time, he can use the domain name without day. If the payment continues, the domain name can continue to be used by the registrant. The intellectual property laws of all countries clearly define the period of validity of the copyright, patent and trademark rights. Beyond the date of expiry, public can use the domain name, so the timeliness of domain name is weaker than traditional intellectual property right.

Third is the breakthrough in uniqueness and specificity. To ensure the identity of domain name, communication protocols in each country specify that every computer on the Internet has unique address, therefore, the domain name has a very strong specificity. After domain name registration, it is absolutely exclusive. However, the specificity of traditional intellectual property right is relatively weak. For example, different rights' holders can apply for the same trademark or trade name in different countries, and they can enjoy the trademark right and the trade name right respectively. 


\section{Conclusion}

Domain name has both technical attributes and business identity attributes which can bring huge economic benefits for the holders. Domain name legal system hasn't been established in China while the laws of traditional intellectual property do not apply to solving the dispute of the domain name. Domain name should be viewed as a new type of independent intellectual property right that different from the traditional intellectual property rights which is a breakthrough and development of it.

\section{Acknowledgements}

This research is supported by The philosophy and Social Science Founding of Jiangsu Province (Project No.16FXB002) and Nanjing university of Chinese medicine.(Project No. ZYWH2017-16)

\section{References}

[1] Chen Jiang,\&Deng Jiong,Anti-cybersquatting consumer protection Act(version),Electronic Intellectual Property Rights, Vol. 2000(8) 22.

[2] Tang Guang-Liang(2012),Domain name disputes and solutions in Internet, fourth volumes of Intellectual property collections-Edited by Zheng Cheng-si,Peking:China University of Political Science and Law Press, pp. 102.

[3] Liu Jin-Lin,Protection of intellectual property on domain name in law view,Peking:China University of Political Science and Law Press, pp. 88-90. 